\title{
A comparison between the preview long hair technique and the classic hair transplant technique
}

\author{
Márcio Crisóstomo, MD Fortaleza,Brazil marcio@implantecapilar.med.br
}

\section{Introduction}

The classic hair transplant technique involves shaving hair from the donor area and having it transplanted while short to the bald area. The patient must wait 6-12 months to see the surgery's result, which in some cases may create great anxiety both for the patient and the surgeon.'

The long hair transplant was first mentioned by Bouhanna in $1989 .^{2}$ In 2006, Brazilian plastic surgeon Dr. Marcelo Pitchon published the preview long hair (PLH) technique, which involves transplanting hair in its natural length and allows for the probable result to be visualized immediately following the surgery (Figure 1). ${ }^{3}$ This technique has been adopted by a number of authors since then..$^{4,5}$

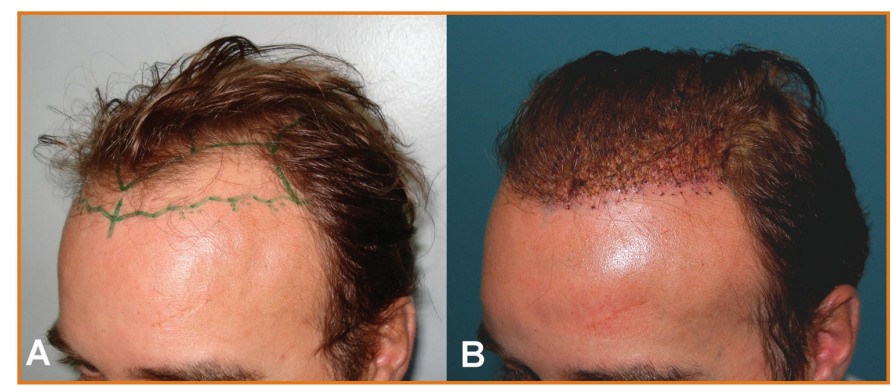

Figure 1. Long hair transplant. A: Marking of anterior hairline; B: appearance immediately after surgery.

In addition to seeing the immediate results, the following are advantages mentioned by Dr. Pitchon: 1) this method allows the surgeon to assess during the surgery whether the amount of transplanted hair is sufficient to cover the intended area and adjustments can be made wherever necessary; 2) the preparation of white hair is easier, so there is less transection and a lower probability of implanting hair in the opposite direction, which would cause inclusion cysts; 3 ) thicker and non-aesthetic follicular units are more easily avoided at the anterior hairline; and 4) the crusts are covered by longer hair and therefore the patient stays away from his or her usual activities for a shorter time. ${ }^{3}$

The aim of this research was to compare the classic hair transplant technique with the PLH technique, taking into account technical aspects pertaining to the surgical procedure as well as the post-operative period and final results.

\section{Method}

A prospective study was carried out with 20 male patients who underwent surgery between October 2008 and January 2009. Ten patients underwent the PLH technique (PLH group) and 10 the classic technique (CT group).

Both groups had a similar average age -43.5 for the TFL group and 40.6 for the CT group, $t$ test $(p=0.59)$. Both groups had an equal distribution according to the Hamilton/Norwood baldness classification.

The classic technique involved shaving the donor area with an electric razor (Figure 2A). The PLH technique in- volved trimming the donor hair until it was 4-5 centimeters long (Figure 2B). Follicular unit (FU) preparation, conservation, and implantation were carried out following a similar procedure with the difference being that the hair was longer in the PLH group (Figure 3). It is important when using the PLH technique to exercise care not to pull implanted long hair and to constantly wash the area in order to prevent blood from accumulating.

The following variables were analyzed and compared: 1 ) time spent removing the donor strip; 2) time spent implanting FUs; 3) total surgery time; 4) total FUs obtained per $\mathrm{cm}^{2}$ of donor area; 5) number of implanted FUs; 6) speed of implantation (FU/min); 7) time until crusts were shed;

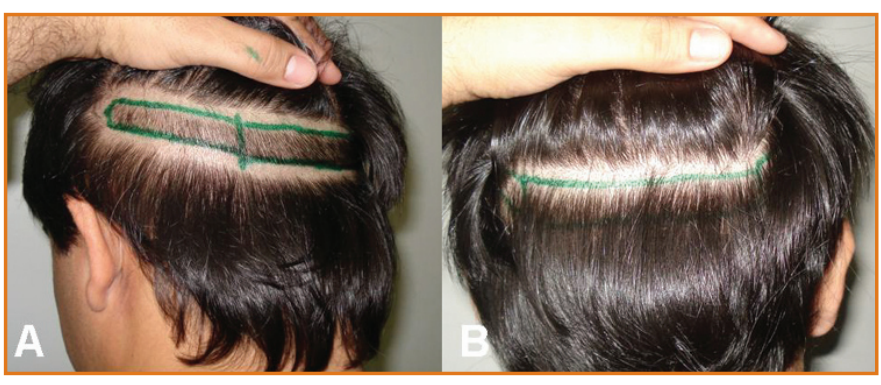

Figure 2. A: Marking of donor area for surgery according to the classic technique; B: marking of donor area with hair maintained in its natural length for surgery according to the PLH technique.

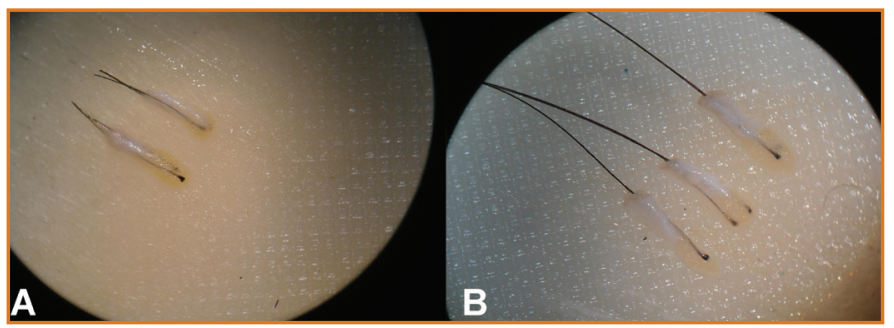

Figure 3. A: Microscopic view of follicular units of shaved hair $(10 \times$ enlarged); B: microscopic view of follicular units of long hair $(10 \times$ enlarged $)$



Figure 4. Surgery times for both groups (donor area harvesting, FU implant, and total surgery time), minutes (mean \pm standard deviation). 
8) time until patients returned to their social activities; and 9) assessment of result after 6 months and, for the PLH technique, a comparison of the 6-month result with the immediate result.

Normal curves were determined for all variables; the sample mean and standard deviation (SD) were also calculated. Student's $t$ test and the respective probabilities were calculated to compare the means for both groups.

\section{Results}

The surgery took longer for the PLH group than the CT group, both in terms of harvesting the donor area as well as the time spent implanting FUs and the surgery's total duration. The difference in the three variables was statistically significant (Figure 4). The average speed of implantation was significantly faster for the CT group (Figure 5).

The average donor area of patients from the PLH group was $32.1 \mathrm{~cm}^{2}(\mathrm{SD} \pm 5.5)$, which was significantly larger than that of the CT group of $26.3 \mathrm{~cm}^{2}(S D \pm 3.6)(p=0.05)$. The average hair density (number of FUs produced per $\mathrm{cm}^{2}$ of donor area) obtained in the PLH group was $54.3 \mathrm{FU} / \mathrm{cm}^{2}$ $(\mathrm{SD} \pm 12.3)$; in the $\mathrm{CT}$ group it was $63.8 \mathrm{FU} / \mathrm{cm}^{2}(\mathrm{SD} \pm 6)$. This difference was not statistically significant $(p=0.08)$. The average number of implanted FUs in patients of the PLH group was $1,717(\mathrm{SD} \pm 258)$, and in the CT group it

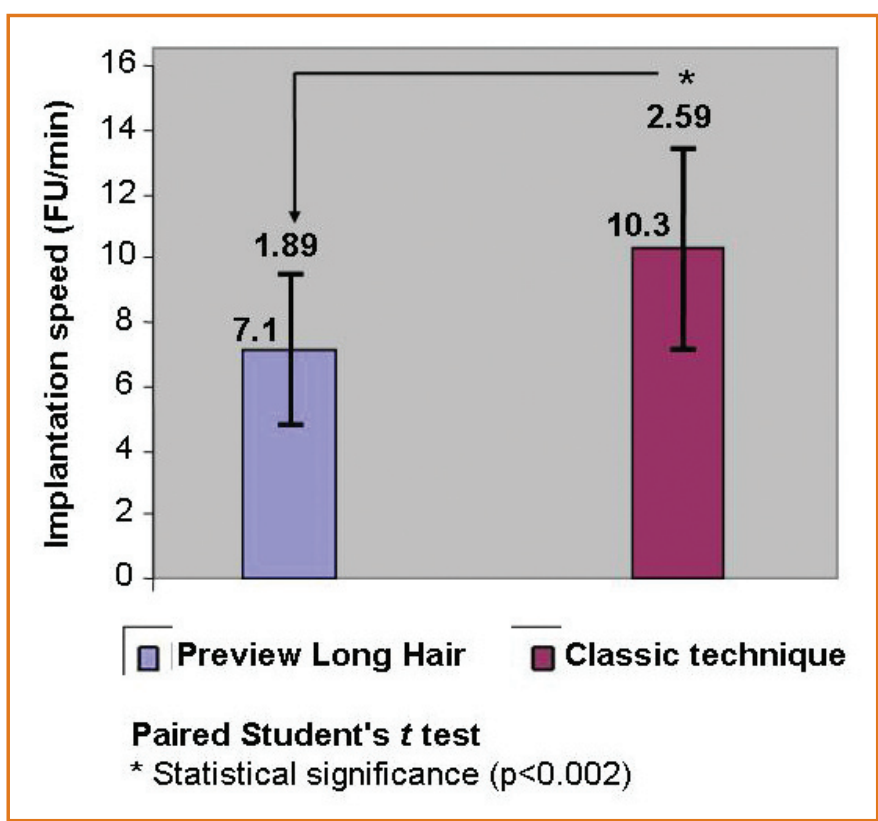

Figure 5. A comparison between speeds of implant (FU/min) for both groups (mean \pm standard deviation).
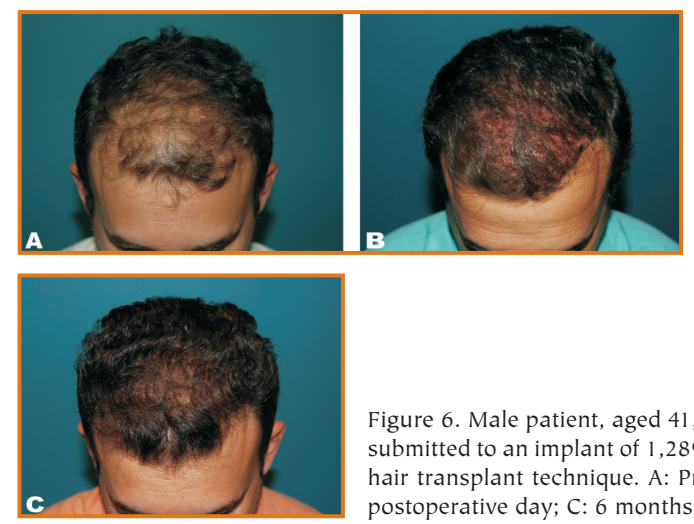

Figure 6. Male patient, aged 41 , class IV baldness, submitted to an implant of 1,289 FU using the long hair transplant technique. A: Preoperative; B: first postoperative day; C: 6 months post-operative. was $1,663(\mathrm{SD} \pm 155)$. This difference was not statistically significant $(p=0.66)$.

It took longer for crusts to shed in the PLH group (14.3 days, $\mathrm{SD} \pm 3.5)$ than in the $\mathrm{CT}$ group $(10.4$ days, $\mathrm{SD} \pm 3.1)$ $(p<0.05)$. Patients from the PLH group returned to their social activities in less time than patients from the CT group (Table 1).

\begin{tabular}{|l|c|c|c|}
\hline \multicolumn{4}{|c|}{ Time to Return to Social Activities } \\
\hline \multirow{3}{*}{ Preview Long Hair } & $<7$ days & $7-14$ days & $>14$ days \\
\cline { 2 - 4 } & 4 & 6 & 0 \\
\hline Classic Technique & 2 & 5 & 3 \\
\hline
\end{tabular}

Table 1. Time elapsed after surgery until patients returned to their social activities.

The similarity between the result obtained on the first day and that obtained after 6 months by the PLH group was deemed to be higher than $80 \%$ by 8 patients and between $60 \%$ and $80 \%$ by the remaining patients (Figures 6 and 7 ). Of the PLH group, 7 believed it was a positive thing to be able to preview the result, 3 said they were indifferent to it, and none believed it was a negative.
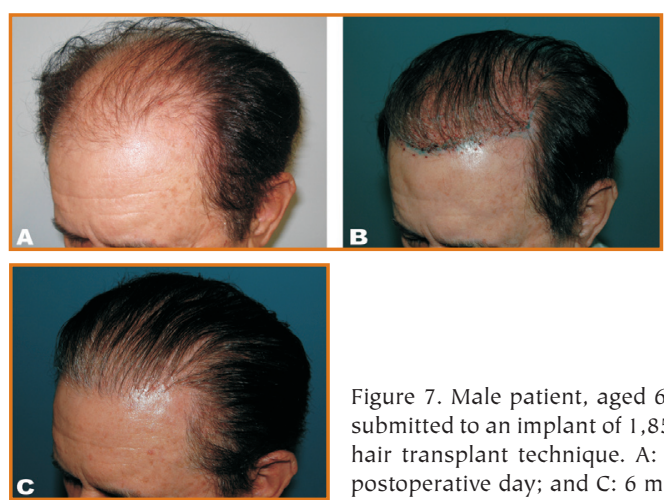

Figure 7. Male patient, aged 68 , class VI baldness, submitted to an implant of 1,856 FUs using the long hair transplant technique. A: Preoperative; B: first postoperative day; and C: 6 months postoperative.

The assessment of results after 6 months made by patients from both groups after analyzing the pre- and postoperative photographs was the same (Table 2).

\begin{tabular}{|l|c|c|c|c|}
\hline & Excellent & Good & Average & Poor \\
\hline Preview Long Hair & 7 & 3 & 0 & 0 \\
\hline Classic Technique & 7 & 3 & 0 & 0 \\
\hline
\end{tabular}

Table 2. Subjective assessment made by patients from both groups regarding the result obtained after 6 months.

\section{Discussion}

The long hair transplant, or PLH, is a breakthrough because it transforms hair transplant surgery, which traditionally requires patience to verify the final result, into a procedure that allows for a preview of this result. ${ }^{3}$

In this study, $70 \%$ of patients who were submitted to the PLH technique saw as positive the fact that they could have an idea of what the later result would be. Eight of 10 patients from the PLH group believed that the result obtained 6 months after the surgery was over $80 \%$ of that seen on the first day. The patients also mentioned that this preview allowed them to have a realistic expectation of what would be obtained from the surgery, and therefore, it was easier to understand the need for a second procedure to increase hair density, if necessary. 
Preview long hair technique

from page 117

Lowering anxiety levels would not be enough to change a well-established surgical routine if there were any negative consequences to the final result. Such a negative effect might be predicted if there was a decrease in the number of FUs implanted, or an excessive increase of surgery time that led to a longer period of ischemia to the grafts. ${ }^{6}$

In this study, there was no decrease in the number of hairs transplanted using the PLH technique.

The speed of implantation was significantly faster for patients who were in the TC group $(p<0.002)$. The PLH technique, however, requires greater care at implantation so that hairs do not get tangled with those already implanted and to avoid pulling them accidentally and consequently extruding the FU from its recipient site. ${ }^{4,5}$ The hair must be washed with saline solution and combed to remove blood several times during the PLH procedure. Such care aims to prevent hairs from being stuck together because of clots, which makes it easier for them to get pulled accidentally, and this may have contributed to the increased surgery time seen in our study.

The time spent implanting FUs for the PLH group was significantly longer than for the CT group. Time spent removing the FUs was also longer. As a result, the PLH group's total duration was significantly longer $(\mathrm{p}<0.0006)$; however, a 6-hour time limit was never exceeded.

In the post-operative period, the formation of inclusion cysts was determined less for patients who were in the PLH group since visualizing the long hair practically excludes the possibility of implanting hairs backwards, which is one of the causes of such cysts. ${ }^{3}$

The visibility during the period in which crusts appear is lower when using the PLH technique because the long hair offers a natural camouflage. This makes that period less perceptible (Figures 6 and 7). ${ }^{3}$ Although it took longer for crusts to shed for patients in the PLH group, it was noted that patients in this group returned sooner to their usual activities. This was probably due to the above-mentioned camouflage effect.

An important piece of information obtained was that the patients' assessment of the result achieved with both techniques was the same. This shows that the final result was not affected by the choice of surgical technique and that the choice between using the classic technique or the preview long hair technique can be made by the surgeon and patient without any consequence to the final result.

\section{Conclusion}

Surgery time was longer for the PLH group due to a longer time spent harvesting the donor area, but mainly from a longer time spent implanting the follicular units. The surgery's total duration did not exceed 6 hours for any of the patients, and the number of follicular units was similar for both groups. The anxiety of waiting to see the result was lower for patients in the PLH group, and the assessment of the final aesthetic result was considered equal for both techniques.

\section{References}

1. Uebel, C.O., ed. Hair restoration: micrografts and flaps. São Paulo, Brazil: OESP Grafica; 2001.

2. Bouhanna, P. Greffes a cheveux longs immediats. Nouv Dermatol. 1989; 8(4):418-420.

3. Pitchon, M. Preview long-hair follicular unit transplantation: an immediate temporary vision of the best possible final result. Hair Transplant Forum Int'l. 2006; 16(4):113115.

4. Bertram, N.G. A simple do it yourself tool to prevent tangling of grafts in long hair transplantation. Hair Transplant Forum Int'l. 2009; 19(4):133-134.

5. Crisóstomo, M.R., et al. Comparação entre a técnica clássica de transplante de unidades foliculares e o transplante de fios longos (preview long hair). Rev Bras Cir Plast. 2010; 25(1):11 7-126.

6. Greco, J.F., R.D. Kramer, and G.D. Reynolds. A "crush study" review of micrograft survival. Dermatol Surg. 1997; 23(9):752-755. ^

\section{A note from Dr. Marcelo Pitchon \\ Belo Horizonte, Brazil}

Dr. Crisóstomo deserves compliments for his beautiful study comparing the preview long hair (PLH) technique and the classic technique. Many other Brazilian and nonBrazilian surgeons have learned the long hair technique and are now performing it in $100 \%$ of their cases, as I have been doing since my very first patient in 2004. Every new surgeon performing the PLH technique is adding personal experience from his or her own background with the conventional technique. Variations include stick-and-place; pre-made incisions; mixed styles; four- or two-handed insertion; local anesthesia or sedation; small, medium, or large sessions; coronal or sagittal, etc. The personal comparison that each surgeon perceives between PLH and the classic conventional technique will be different from that of others according to experience, ability, and the efficacy of personal style to achieve the main objectives of this technique, which are fascination of the patient and enhancing patients' perception of the type of quality hair transplant surgery achieved by our field. (I am considering here objectives evaluated from the patients' perspective and not the technical advantages that patients cannot compare, such as the visual definition by the surgeon of the ideal density and hence optimization of the donor resource.) Issues like the incidence of tangling and extrusion of grafts caused by bleeding, clots, and other factors may vary considerably from surgeon to surgeon being influenced by his particular way of working. The increased duration of procedure issues has always been present in every significant scientific advance in our field (e.g., microscopic dissection) and, with time, has ceased to be an issue. Technical individual differences between surgeons' styles can make the comparison between two techniques in any study's specific surgical environment valid, yet almost impossible to generalize to a broader universe of surgeons utilizing the PLH concepts or to the PLH methodology itself. Although Dr. Crisóstomo has reinforced and correctly alerted us to some important details regarding the care necessary to deal with long hair grafts, his study confirms many, if not all, the findings 
reported in the 2006 article, "Preview long hair follicular unit transplantation: an immediate temporary vision of the best possible final result" (Pitchon, M. Hair Transplant Forum Int'l. 2006; 16(4):113) and stimulates other surgeons to experience for themselves the pleasure of giving patients the PLH technique. I am sure that if, in a future study, we could include a group of patients who were previously submitted to the conventional technique and would now be submitted to the PLH technique, we would probably have this group totally or almost totally in favor of the PLH method.

The PLH concept and technique were developed in order to show the current state of hair transplantation excellence. It was originally conceived to show, but not only show; to show immediately, how beautiful, emotional, artistic and fascinating today's hair transplantation is. It is very difficult to compare the PLH technique with the classic one because objective comparisons do not take the above main subjective elements into account in a measurable way. To perform PLH in the totality of its conception, these and other subjective elements are essential, and obviously associated with the objective and scientific ones. As an example, in order to show results on the same day of surgery (without a dressing to hide them), you must have a beautiful bloodless post-operative field (also intraoperative, if possible) and incisions that perfectly fit the grafts so that they do not bleed easily and stay firm and difficult to remove, no matter if the PLH or CT technique was used. After surgery the hair should always be well cleaned and parted the way the patient likes because it provokes patients' positive reactions and emotions. So if the surgeon performing the conventional technique makes incisions too big for the grafts, he will first have to make a move into customized incisions in order to step toward PLH. The PLH technique has a learning curve that started for me before its conception in 2004, I am referring to a curve focused on improving the classic technique as much as possible before a novice to PLH starts performing it. Thus, I think that the extra time spent at the beginning of the PLH learning curve is also due to details that are not exclusively inherent to PLH, but rather to the improvement of the classic technique itself. Just leaving the hair longer, without an extremely refined technique, does not mean that you can show and provide to the patient an artistic preview, ending in the patient's fascination. That could be a disaster. One can leave longer hair even in gigantic old grafts inserted into big incisions or punch holes, but this is not something you can show to patients in order to fascinate them and promote the perception of quality in our field, two of the most essential elements of the creation of preview long hair follicular unit transplantation.

One last point: In my opinion, for a case to qualify as a "preview" long hair transplant the donor hair needs to be any length longer than shaved, whether $5 \mathrm{~mm}$ or $15 \mathrm{~mm}$; any length longer than the height or diameter of the crusts (if they exist), since the surgeon's objective is to produce a preview for the patient. The expression "long hair" was used in the original paper so that the medical community could differentiate it from shaved hair, the current technique at that time, in 2004. The main idea centers around the "preview" concept, and the length of the hair is just a means of achieving that preview.

\section{ILTER \\ Scaping the next generation of FUE professionals. \\ 4-9th of October 2010}

\section{FUE Medical Training Course for Doctors}

Hands on training and medical understanding of FUE. Surgical training in FUE with Dr. Iter

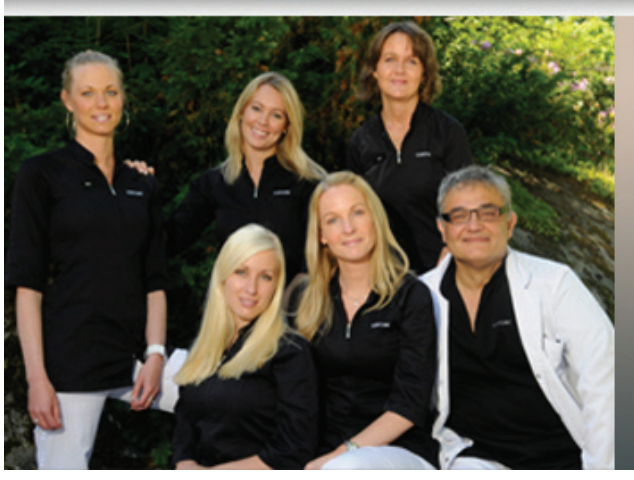

More info
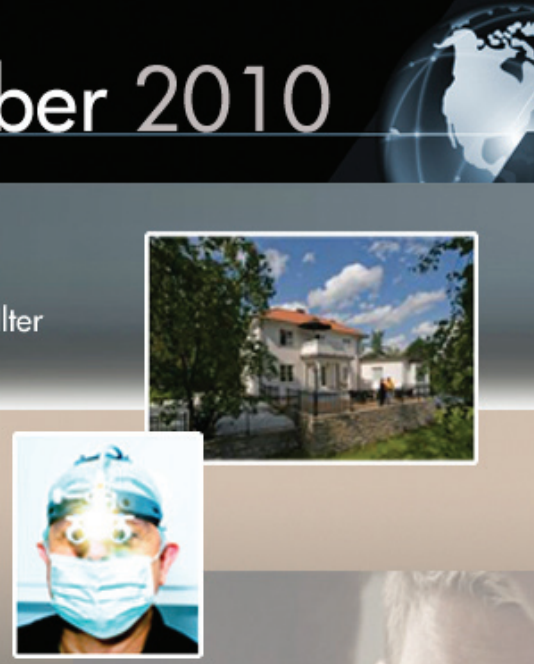